\title{
Vowel Contrast and Speech Intelligibility in Dysarthria
}

\author{
Heejin Kim ${ }^{\mathrm{a}}$ Mark Hasegawa-Johnson ${ }^{\mathrm{b}}$ Adrienne Perlman ${ }^{\mathrm{c}}$ \\ ${ }^{a}$ Beckman Institute and Departments of ${ }^{b}$ Electrical and Computer Engineering and ${ }^{\circ}$ Speech and Hearing Science, \\ University of Illinois, Urbana-Champaign, III., USA
}

\section{Key Words}

Dysarthria $\cdot$ Vowel contrast $\cdot$ Speech intelligibility

\begin{abstract}
Background/Aims: This study examined the spectral characteristics of American English vowels in dysarthria associated with cerebral palsy (CP), and investigated the relationship between a speaker's overall speech intelligibility and vowel contrast. Methods: The data were collected from 12 American English native speakers ( 9 speakers with a diagnosis of $C P$ and 3 controls). Primary measures were $F_{1}$ and $F_{2}$ frequencies of 3 corner vowels $/ i, a, u /$ and 3 noncorner vowels $/ I, \varepsilon, v /$. Six acoustic variables were derived from the formant measures, and were regressed against intelligibility: corner vowel space, noncorner vowel space, mean distance between vowels, $F_{1}$ and $F_{2}$ variability, and overlap degree among vowels. Results: First, the effect of vowel was significant for both $F_{1}$ and $F_{2}$ measures for all speakers, but post hoc analysis revealed a reduced distinction at lower intelligibility. Second, regression functions relating intelligibility and acoustic variables were significant for overlap degree among vowels, $F_{1}$ variability, corner vowel space and mean distance between vowels. Overlap degree among vowels accounted for the greatest amount of variance in intelligibility scores. Conclusion: A speaker's overall intelligibility in dysarthric speech is better represented by the overlap degree among vowels than by the vowel space.
\end{abstract}

Copyright ๑ 2010 S. Karger AG, Basel

\section{Introduction}

Dysarthria is often characterized by imprecise articulation of segments. Previous studies have noted vowel centralization and reduced vowel space as the common characteristics of vowels in dysarthric speech [1-9]. However, the strength of the relationship between intelligibility and the vowel space area varied widely across studies, ranging from 0 to $71 \%$. For example, vowel space area accounted for about $46 \%$ of the variance in intelligibility scores for speakers with amyotrophic lateral sclerosis (ALS) or Parkinson's disease (PD) [3], and 63-68\% for Mandarin-speaking adults with cerebral palsy (CP) [4]. On the other hand, the strength was only $6-8 \%$ for speakers with PD or multiple sclerosis [5], and $0 \%$ for speakers with ALS [6]. The relationship between vowel space and intelligibility in dysarthric speech has been of interest to researchers and clinicians, particularly for the purpose of evaluating speaking rate modification as a treatment technique to enhance vowel contrast and thereby improve a speaker's overall speech intelligibility [5-8].

In addition to vowel space areas, researchers have examined the mean distance between vowels to quantify the degree of vowel dispersion in the vowel space, but this method was mostly used in normal speech studies [1011]. For example, Smiljanic and Bradlow [10] measured the distance of each of 3 vowels /i, a, u/ from the central point in the speaker's $F_{1} \times F_{2}$ vowel space and reported a greater mean distance in clear speech compared to con-

\section{KARGER}

Fax +4161306 1234 E-Mail karger@karger.ch www.karger.com
() 2010 S. Karger AG, Basel
Heejin Kim

Beckman Institute for Advanced Science and Technology

University of Illinois at Urbana-Champaign

405 North Mathews Avenue, Urbana, IL 61801 (USA)

Tel. +1 217244 4972, Fax +1 217333 2922, E-Mail hkim17@illinois.edu 
versational speech. On the other hand, Neel [11] found that neither mean distance nor vowel space area predicted more than $12 \%$ of the variance in vowel identification scores in normal speech. Several studies compared the formant values of contrastive vowels in dysarthric speech, focusing on the perception of vowel contrast and its acoustic correlates. Acoustic differences were reported between vowel pairs such as front-back, high-low and tense-lax produced by speakers with CP [12]. However, such differences were not necessarily associated with higher intelligibility, suggesting that while the speakers produced the vowel contrast by acoustic differentiation, it varied from normal speech. A different finding was reported for speakers with ALS, PD and cerebrovascular accident [13]: vowels were perceived as different despite a larger degree of overlap between high versus low vowels.

In pursuing research on speech intelligibility and its relation to vowel contrast in dysarthria, questions remain concerning the following issues. First, the size of acoustic vowel space has been a primary measure in previous research on dysarthric vowels. Although vowels in a larger vowel space are expected to be more distinct from one another, the size of the vowel space alone does not address whether, and to what extent, vowel distinctiveness is maintained. Second, most research on dysarthric vowel space has focused on corner vowel space formed by vowels such as /i, a, u, æ/, leaving it unclear whether the extent of articulatory imprecision in dysarthric speech is uniform across different vowel categories. Tjaden et al. [14] is the only study on noncorner vowel space in dysarthric speech. Specifically, they examined the noncorner vowel space formed by lax vowels $/ \mathrm{I}, \varepsilon, \mho /$ at different speaking rates for speakers with ALS or PD. When compared to control speakers, a large degree of speaker variation was found for speakers with dysarthria. However, lax vowel space areas were significantly smaller for speakers with ALS, but not for speakers with PD. As the authors stated, it was not clear whether the different findings for the ALS versus $\mathrm{PD}$ groups were due to differences in the type or severity levels of dysarthria. Aiming to address the relation between dysarthria severity and vowel production, this study examined the relationship between speech intelligibility and the spectral characteristics of American English vowels in spastic dysarthria associated with CP. The following research questions were addressed: (1) are vowel contrasts reduced at a lower intelligibility level, (2) which acoustic variable of vowels is most strongly correlated with intelligibility, and (3) are corner versus noncorner vowels differently related to intelligibility?

\section{Materials and Methods}

\section{Participants}

The data were collected from 12 American English native speakers composed of 9 speakers with a diagnosis of CP (8 males and 1 female) and 3 male control speakers, ranging in age from 18 to 58 years. In the CP group, 8 speakers reported a diagnosis of principally spastic dysarthria, and 1 speaker reported a diagnosis of mixed spastic and athetoid dysarthria. Their intelligibility scores ranged from 2 to $95 \%$, which were estimated on the basis of word transcription tasks conducted by naive listeners: the method is described below in detail. The control speakers reported no history of speech or language disability, and their intelligibility scores ranged from 96 to $98 \%$.

\section{Recording Procedure and Materials}

The materials analyzed for this study were a subset of the database collected under the project for developing automatic speech recognition systems for dysarthric speech [15]. Speech was recorded using an 8-microphone array, which was previously developed for the AVICAR (Audio-Visual Speech in a Car) project [16]. The array was mounted on a laptop computer screen during recording. Speech was recorded via an 8-channel Firewire audio interface (MOTU $828 \mathrm{mkII}$ ), with a sampling rate of $48 \mathrm{kHz}$. The speakers sat comfortably in front of the laptop computer and were asked to read a word displayed on a PowerPoint slide on the laptop monitor. Each speaker produced 3 blocks of 255 real words, totaling 765 words. Each block contained 5 word categories: digits (e.g. zero, one), computer commands (e.g. enter, delete), international radio alphabet letters (e.g. alpha, bravo), 100 common words (e.g. a, the) and 100 uncommon words (e.g. naturalization, exploit). The 100 common words were the most frequently occurring words in the Brown corpus of written English [17]. The 100 uncommon words were selected from children's novels digitized by Project Gutenberg [18], using an algorithm that allowed for recording of all possible sequences of phones in American English. Speakers were always given a break between blocks and were allowed additional breaks as needed.

\section{Intelligibility Rating}

Each speaker's speech was rated for intelligibility for the purpose of acquiring an index of dysarthria severity. Five naive listeners were recruited for each speaker, totaling 60 listeners (5 listeners $\times 12$ speakers). Inclusion criteria for listeners were that they should (a) be between 18 and 40 years old, (b) be native speakers of American English, (c) have no identified language disability, (d) have had no more than incidental experience with persons having speech disorders, and (e) have had no training in phonetic transcription. A transcription task was selected for the intelligibility rating because it has been suggested to be more valid, objective and consistent compared to subjective scaling (e.g. equalappearing interval scaling and direct magnitude estimates), particularly when a large number of samples are rated $[19,20]$.

The listeners were informed that they would be listening to real words in American English produced by an individual with a speech disorder, and were asked to provide orthographic transcriptions of each word. They were also asked to indicate the degree of certainty about their word choice by writing a number (0-2) next to each word: ' 0 ' for 'not sure at all', ' 1 ' for 'somewhat sure' and '2' for 'completely sure'. They were allowed to listen to 
words as many times as needed, but were asked not to go back to previous words that had already been transcribed. Each listener transcribed a list of 225 words: 200 distinct words from the second-block recording. For intralistener reliability assessment, 25 words were arbitrarily selected and repeated twice. The speech files were randomly ordered, with the constraint that repeated words should not be adjacent to one another. The percentage of correct responses was calculated per listener, and averaged across the 5 listeners who had transcribed the same speaker's data. Each speaker's intelligibility was classified into one of 4 nonoverlapping categories, which were operationally defined as very low (0$25 \%)$, low (26-50\%), mid (51-75\%) and high (76-100\%). For all listeners, the intralistener reliability score was $91.64 \%$ on average for the words marked ' 2 ' (i.e. words that listeners were completely sure of). For words marked ' 1 ' or ' 0 ', the 2 transcriptions were either identical or phonetically similar to each other.

\section{Acoustic and Statistic Analysis}

The 3 corner vowels /i, a, u/ and 3 noncorner vowels /I, $\varepsilon, \mathrm{U} /$ were selected from stressed syllables in the categories of digits, computer commands, radio alphabet and common words in all 3 blocks. Nine uncommon words that contained / $\mathrm{u} /$ were added to the data set to ensure a minimum of 12 occurrences of each vowel per speaker. Vowels that were preceded or followed by $/ \mathrm{r} /, / 1 /$, $/ \mathrm{w} /$ or $/ \mathrm{j} /$ were excluded. A total of 126 vowels were studied per speaker: 21 occurrences of /i/, 15 of /a/, 12 of /u/, 39 of /I/, 27 of / $\varepsilon /$ and $12 \mathrm{of} / \mathrm{v}$. The temporal midpoint of the steady portion of the vowel was manually labeled via visual observation of the waveform and wideband spectrogram in Praat [21]. $\mathrm{F}_{1}$ and $\mathrm{F}_{2}$ values were extracted in bark units using a custom-written script, wherein formant values were estimated by the Burg algorithm with a 25 -ms window. This algorithm was constrained by a specified ceiling of the formant search range and a specified maximum number of formants for each vowel. The maximum number of formants was always set to 5 . The ceiling of the formant search range was typically $5,000 \mathrm{~Hz}$ for male control speakers. For speakers with $\mathrm{CP}$, it varied from 4,000 to $5,000 \mathrm{~Hz}$ depending on the backness of vowels. Tokens were excluded from our analysis if their formant values were outside the range of \pm 3 standard deviations.

The first analysis examined the vowel contrast for each speaker individually, based on nonparametric Kruskal-Wallis tests with the dependent measure ' $\mathrm{F}_{1}$ and $\mathrm{F}_{2}$ frequencies' and the independent factor 'vowel'. Nonparametric tests were used because many of the vowel groups being compared were of unequal variance according to Levene's test for homogeneity of variance. When the Kruskal-Wallis tests identified significant differences among vowels, post hoc analyses were performed to determine which vowel means differed. Nonparametric Dunnett $C$ tests (pairwise multiple comparisons) were selected due to the unequal variance and the small sample size of the data.

Second, a series of regression analyses was conducted to evaluate the relationship between a speaker's overall intelligibility and the 6 acoustic variables: (1) corner vowel space, (2) noncorner vowel space, (3) mean distance between vowels, (4) $\mathrm{F}_{1}$ variability, (5) $\mathrm{F}_{2}$ variability, and (6) overlap degree among the 6 vowels. Corner vowel space, noncorner vowel space and mean distance between vowels served as indices of the articulatory working space of vowels and vowel space dispersion. $F_{1}$ and $F_{2}$ variability was examined to assess the relative acoustic stability in achieving vowel targets. The degree of overlap among the 6 vowels was measured to discover the extent to which a vowel encroaches neighboring vowels.

Corner vowel space was defined as the euclidean area of a triangle defined by the $\mathrm{F}_{1}$ and $\mathrm{F}_{2}$ values of the $/ \mathrm{i}, \mathrm{a}, \mathrm{u} /$ vowels. The size was calculated using the equation $\Delta=1 / 2\left(-x_{2} y_{1}+x_{3} y_{1}+\right.$ $\left.x_{1} y_{2}-x_{3} y_{2}-x_{1} y_{3}+x_{2} y_{3}\right)$, where $x_{1}, x_{2}, x_{3}=$ mean $\mathrm{F}_{1}$ values, and $y_{1}$, $y_{2}, y_{3}=$ mean $\mathrm{F}_{2}$ values of each of the corner vowels. Likewise, the size of the noncorner vowel space was calculated using mean $\mathrm{F}_{1}$ and $F_{2}$ values of the $/ \mathrm{I}, \varepsilon, \mathrm{U} /$ vowels. Variability in $\mathrm{F}_{1}$ and $\mathrm{F}_{2}$ was obtained for each speaker by averaging the standard deviations of the $F_{1}$ and $F_{2}$ values, respectively, for the 6 vowels. The mean distance between vowels was measured for each speaker by calculating the distance between the 2 neighboring vowels in the $F_{1} \times F_{2}$ space (i.e. the distance of each of the following 6 pairs: $/ \mathrm{i} /-/ \mathrm{I} /$, $/ \mathrm{I} /-/ \varepsilon /, / \varepsilon /-/ \mathrm{a} /, / \mathrm{a} /-/ \mathrm{v} /, / \mathrm{u} /-/ \mathrm{u} /$ and $/ \mathrm{i} /-/ \mathrm{u} /)$, and by averaging these 6 distances. The overlap degree among vowels was estimated using quadratic discriminant analysis in Matlab ${ }^{\mathrm{TM}}$ (release 2006a). First, each speaker's data were divided into 3 sets with the constraint that each set should contain the same number of occurrences of the same vowel phoneme. Discriminant analyses were performed for each speaker using a 3-fold cross-validation process: 3 tests were performed, each one with 2 data sets for training and the remaining one for testing. Each test provided a misclassification error rate (i.e. the percentage of observations that were misclassified due to overlap among vowels). The overlap degree of vowels for each speaker was indexed by the average error rate of the 3 tests. Since our regression analyses involved 6 dependent measures, the $\alpha$ level was divided by 6 , yielding a per-comparison $\alpha$ level of $0.0083(=0.05 / 6)$.

\section{Results}

\section{Vowel Contrast within Speaker}

Table 1 displays the results of the Kruskal-Wallis tests on the $\mathrm{F}_{1}$ measures (bark); control speakers are listed first, and speakers with CP are listed in decreasing order of intelligibility. Analysis revealed a significant main effect of vowel on the $F_{1}$ measures for all speakers. Subsequent post hoc analyses revealed that control speakers (C1-C3) and highly intelligible speakers (CP1, CP2) made at least 4 levels of height distinction: low $(/ a /)$ versus mid $(/ \varepsilon /)$ versus high lax $(/ U, \mathrm{I} /)$ versus high tense $(/ \mathrm{u}, \mathrm{i} /)$. As the intelligibility level decreased to the mid and low categories (CP3, CP4; CP5, CP6), a distinction between mid versus high lax, or between high lax versus high tense was not maintained (e.g., for CP6, the vowel height of / $/$ vs. /u/ was not significantly different, nor was it for /I/ vs. /i/). Two speakers in the very low category (CP8, CP9) exhibited a further reduction in the height distinction by grouping /a, $\varepsilon, v /$.

Table 2 shows the results for the $\mathrm{F}_{2}$ measures (bark); the effect of vowel was significant for all speakers, but post hoc analyses showed a reduced distinction of vow- 
Table 1. Mean $\mathrm{F}_{1}$ values (bark) and results of Kruskal-Wallis and post-hoc analyses

\begin{tabular}{|c|c|c|c|c|c|c|c|c|}
\hline Speaker & i & I & $a$ & $\varepsilon$ & $\mathrm{u}$ & U & Kruskal-Wallis & Post hoc \\
\hline $\mathrm{C} 1$ & 3.58 & 4.52 & 6.85 & 5.4 & 3.87 & 4.82 & 0.000 & $a>\varepsilon>U>I>u>i$ \\
\hline $\mathrm{C} 2$ & 3.56 & 4.59 & 7.27 & 6.17 & 3.71 & 4.87 & 0.000 & $\mathrm{a}>\varepsilon>U>\mathrm{I}>\mathrm{u}, \mathrm{i}$ \\
\hline $\mathrm{C} 3$ & 3.69 & 4.38 & 6.39 & 5.65 & 3.82 & 4.52 & 0.000 & $a>\varepsilon>U, I>u, i$ \\
\hline $\mathrm{CP} 1$ & 3.44 & 4.65 & 7.23 & 5.5 & 3.8 & 4.72 & 0.000 & $a>\varepsilon>U, I>u>i$ \\
\hline $\mathrm{CP} 2$ & 3.44 & 4.36 & 6.29 & 5.37 & 3.81 & 4.45 & 0.000 & $a>\varepsilon>U, I>u>i$ \\
\hline $\mathrm{CP} 3$ & 3.61 & 4.11 & 5.87 & 4.97 & 3.85 & 4.43 & 0.000 & $\mathrm{a}>\varepsilon>U>\mathrm{I}, \mathrm{u}>\mathrm{u}, \mathrm{i}$ \\
\hline $\mathrm{CP} 4$ & 3.49 & 4.51 & 6.49 & 5.73 & 3.81 & 5.07 & 0.000 & $a>\varepsilon, U>U, I>u>i$ \\
\hline CP5 & 4.06 & 4.8 & 6.85 & 5.42 & 4.52 & 5.08 & 0.000 & $\mathrm{a}>\varepsilon, U>U, \mathrm{I}>\mathrm{I}, \mathrm{u}>\mathrm{i}$ \\
\hline CP6 & 3.08 & 3.46 & 5.63 & 4.09 & 3.55 & 3.84 & 0.000 & $\mathrm{a}>\varepsilon, U>U, \mathrm{u}, \mathrm{I}>\mathrm{I}, \mathrm{i}$ \\
\hline $\mathrm{CP} 7$ & 3.53 & 3.79 & 5.51 & 4.63 & 3.3 & 3.8 & 0.000 & $a>\varepsilon>U, I, i>i, u$ \\
\hline CP8 & 3.87 & 4.22 & 4.98 & 4.66 & 3.98 & 4.34 & 0.000 & $\mathrm{a}, \varepsilon, \mathrm{v}>\mathrm{U}, \mathrm{I}, \mathrm{u}, \mathrm{i}$ \\
\hline CP9 & 3.79 & 4.02 & 4.78 & 4.5 & 3.79 & 3.96 & 0.000 & $\mathrm{a}, \varepsilon, U>U, \mathrm{I}, \mathrm{u}, \mathrm{i}$ \\
\hline
\end{tabular}

$\mathrm{C}=$ Control. $\mathrm{CP}=$ Speakers with $\mathrm{CP}$.

Table 2. Mean $\mathrm{F}_{2}$ values (bark) and results of Kruskal-Wallis and post-hoc analyses

\begin{tabular}{|c|c|c|c|c|c|c|c|c|}
\hline Speaker & i & I & $a$ & $\varepsilon$ & $\mathrm{u}$ & U & Kruskal-Wallis & Post hoc \\
\hline $\mathrm{C} 1$ & 13.33 & 12.29 & 9.93 & 11.29 & 8.33 & 9.97 & 0.000 & $\mathrm{i}>\mathrm{I}>\varepsilon>\mathrm{U}, \mathrm{a}>\mathrm{u}$ \\
\hline $\mathrm{C} 2$ & 13.38 & 12.67 & 10.23 & 11.58 & 9.45 & 10.62 & 0.000 & $\mathrm{i}>\mathrm{I}>\varepsilon>\mathrm{U}, \mathrm{a}>\mathrm{u}$ \\
\hline $\mathrm{C} 3$ & 13.77 & 12.98 & 9.45 & 12.08 & 11.03 & 10.73 & 0.000 & $\mathrm{i}>\mathrm{I}>\varepsilon>\mathrm{u}, \mathrm{U}>\mathrm{a}$ \\
\hline $\mathrm{CP} 1$ & 13.83 & 12.51 & 9.53 & 11.94 & 9.1 & 9.99 & 0.000 & $\mathrm{i}>\mathrm{I}>\varepsilon>\mathrm{U}, \mathrm{a}, \mathrm{u}$ \\
\hline $\mathrm{CP} 2$ & 12.13 & 11.66 & 9.51 & 11.05 & 6.82 & 8.81 & 0.000 & $\mathrm{i}>\mathrm{I}>\varepsilon>\mathrm{a}, \mathrm{U}>\mathrm{u}$ \\
\hline $\mathrm{CP} 3$ & 12.87 & 12.52 & 10.18 & 11.94 & 9.09 & 9.9 & 0.000 & $\mathrm{i}>\mathrm{I}>\varepsilon>\mathrm{a}, \mathrm{U}, \mathrm{u}$ \\
\hline $\mathrm{CP} 4$ & 12.76 & 12.17 & 8.93 & 11.77 & 9.05 & 10.31 & 0.000 & $\mathrm{i}>\mathrm{I}>\varepsilon>\mathrm{U}>\mathrm{u}, \mathrm{a}$ \\
\hline CP5 & 13.2 & 11.74 & 10.21 & 11.41 & 9.55 & 10.46 & 0.000 & $\mathrm{i}>\mathrm{I}, \varepsilon>\mathrm{U}, \mathrm{a}>\mathrm{u}$ \\
\hline CP6 & 12.26 & 11.88 & 9.22 & 11.74 & 8.21 & 9.43 & 0.000 & $\mathrm{i}, \mathrm{I}>\varepsilon>\mathrm{v}, \mathrm{a}>\mathrm{u}$ \\
\hline $\mathrm{CP} 7$ & 11.93 & 12.6 & 9.44 & 11.96 & 9.57 & 9.48 & 0.000 & $\mathrm{I}, \mathrm{i}>\mathrm{i}, \varepsilon>\mathrm{u}, \mathrm{v}, \mathrm{a}$ \\
\hline CP8 & 12.06 & 12.11 & 10.86 & 11.81 & 11.2 & 11.4 & 0.000 & $\mathrm{i}, \mathrm{I}>\varepsilon, \mathrm{v}, \mathrm{u}>\mathrm{v}, \mathrm{u}, \mathrm{a}$ \\
\hline CP9 & 11.25 & 10.96 & 10.13 & 10.9 & 9.57 & 10.13 & 0.000 & $\mathrm{i}, \mathrm{I}, \varepsilon>\mathrm{a}, \mathrm{v}, \mathrm{u}$ \\
\hline
\end{tabular}

els as a speaker's overall intelligibility decreased. The control speakers and CP speakers with higher intelligibility exhibited significantly different $\mathrm{F}_{2}$ values for the $/ \varepsilon /$ vowel both from the front vowels /i, I/ and the back vowels $/ \mathrm{u}, \mathrm{u} /$. On the other hand, as for CP speakers with lower intelligibility, the $/ \varepsilon /$ vowel was not distinct from either the /i/ (speakers CP7 and CP9) or the back vowels (speaker CP8). Figure 1 displays the mean $\mathrm{F}_{1}$ and $\mathrm{F}_{2} \mathrm{CO}-$ ordinates for each speaker. Corner vowel space and noncorner vowel space are separately shown. In general, speakers with higher intelligibility tended to have a larger vowel space compared to speakers with lower intelligibility.

\section{Relationship between Speech Intelligibility and Acoustic Measures of Vowels}

Regression functions relating intelligibility and acoustic measures of vowels were significant for the following measures: corner vowel space $[\mathrm{F}(1,10)=22.052 ; \mathrm{p}=0.001$; $\left.\mathrm{r}^{2}=0.69\right]$, mean distance $[\mathrm{F}(1,10)=22.413 ; \mathrm{p}=0.001$; $\left.\mathrm{r}^{2}=0.69\right], \mathrm{F}_{1}$ variability $[\mathrm{F}(1,10)=28.959 ; \mathrm{p}=0.000 ;$ $\left.\mathrm{r}^{2}=0.74\right]$ and overlap degree $[\mathrm{F}(1,10)=222.822 ; \mathrm{p}=0.000$; $\left.\mathrm{r}^{2}=0.96\right]$. In contrast, the regression functions for noncorner vowel space and $\mathrm{F}_{2}$ variability were not significant. Scatter plots of the 4 significant functions are shown in figure 2. Corner vowel space did not reach an $r^{2}$ value that was equivalent to the overlap degree. Inspection of figure 2 a suggests that the data point of speaker C3 might 


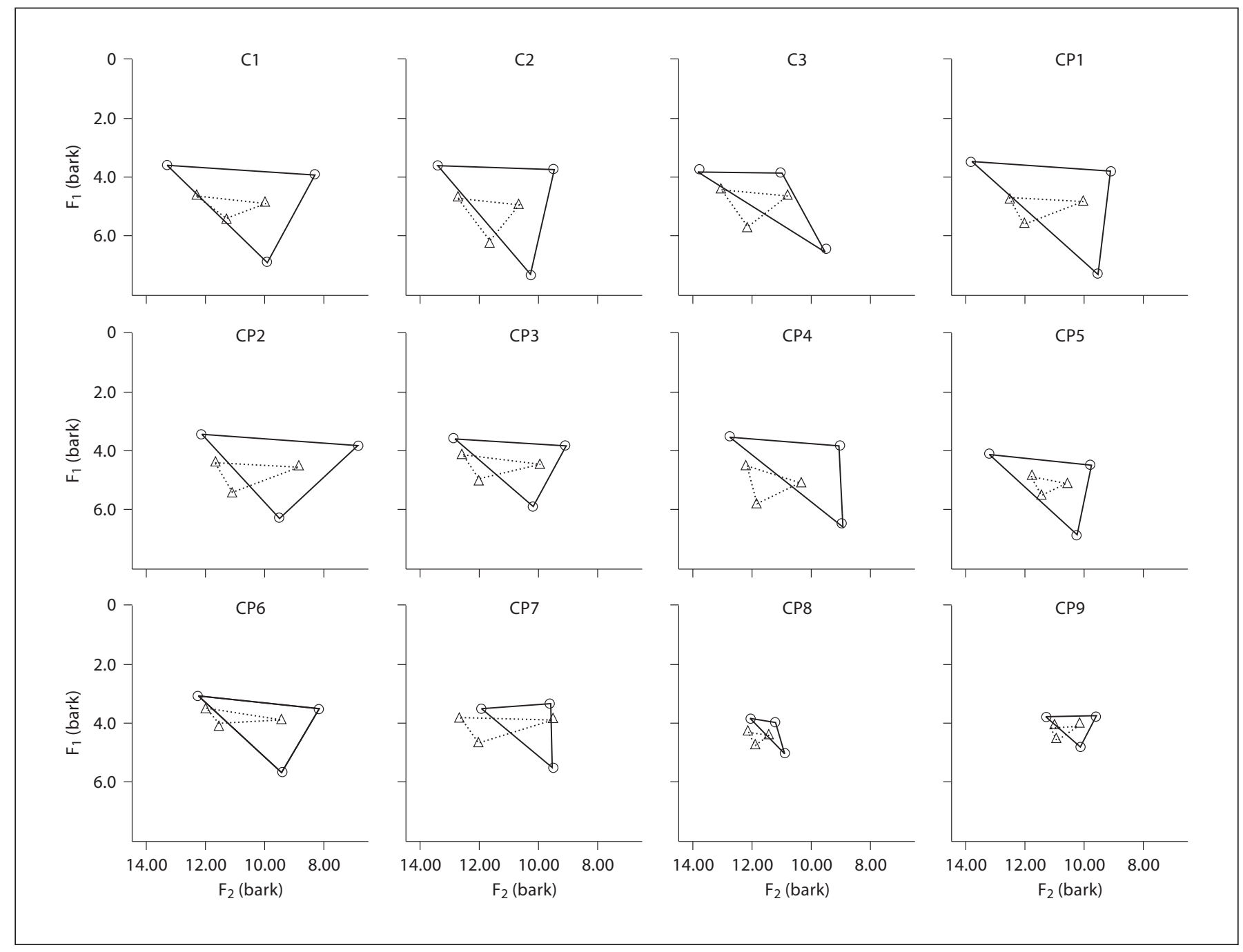

Fig. 1. Mean $F_{1}$ and $F_{2}$ coordinates for each speaker. $\bigcirc=$ Corner vowel space; $\triangle=$ noncorner vowel space.

exert a significant influence on the lay of the regression line between intelligibility and corner vowel space. When the speaker C3 data point was removed, the $\mathrm{r}^{2}$ value increased to 0.90 , confirming that speaker C3 had a relatively small corner vowel space for his intelligibility level. It is also notable that $\mathrm{C} 3$ exhibited the lowest value in $\mathrm{F}_{1}$ variability (fig. 2c) and a considerably low overlap degree (fig. 2d). By contrast, $\mathrm{CP} 1$ had a high $\mathrm{F}_{1}$ variability for his intelligibility level (fig. 2c). When the speaker CP1 data point was removed, the $\mathrm{r}^{2}$ value of $\mathrm{F}_{1}$ variability increased to 0.89 . Despite the high $\mathrm{F}_{1}$ variability, his overlap degree was low (fig. 2d), owing to the fact that CP1 had the largest corner vowel space (fig. 2a), compensating the high variability in $\mathrm{F}_{1}$.

Vowel Contrast and Speech Intelligibility

\section{Discussion}

Our first analysis showed significant main effects of vowel on both $\mathrm{F}_{1}$ and $\mathrm{F}_{2}$ measures for all speakers. This finding suggests that speakers with $\mathrm{CP}$, including those with severely reduced intelligibility, attempt to produce vowels distinctively. The subsequent post hoc comparisons, however, revealed a reduced distinction among vowels both in the $F_{1}$ and $F_{2}$ measures as intelligibility decreased. Second, regression analyses revealed that overlap degree among vowels accounted for the greatest amount of variance in intelligibility scores. $F_{1}$ variability, corner vowel space area, and mean distance among vowels were also significantly related to intelligibility. Spe-

Folia Phoniatr Logop 2011;63:187-194 191 

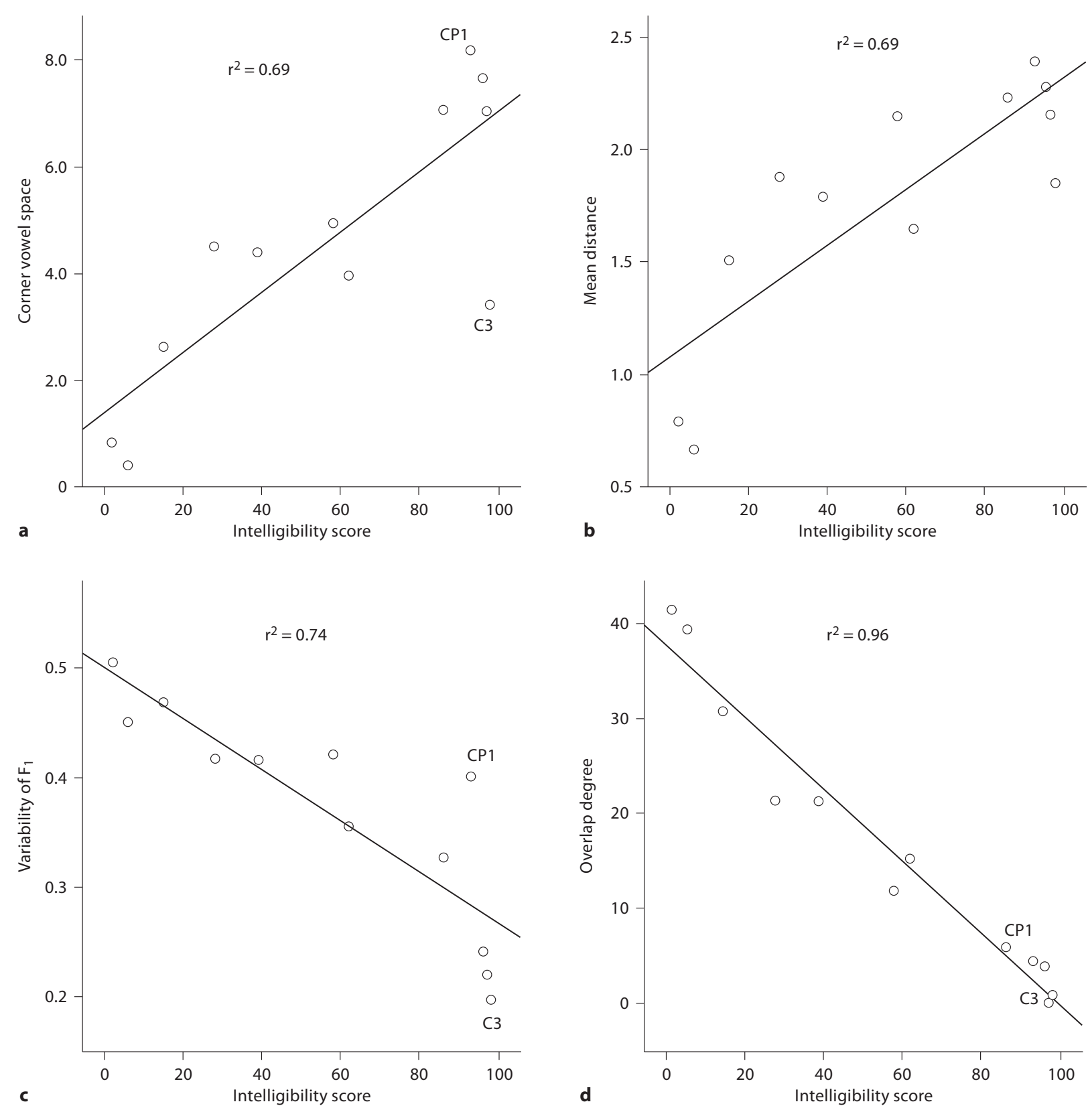

Fig. 2. Scatter plots of the 4 significant functions.

cifically, lower intelligibility was associated with (1) increased overlap among vowels, (2) increased $F_{1}$ variability - i.e. decreased acoustic stability in achieving vowel height -, (3) reduced corner vowel space - i.e. reduced articulatory working space - and (4) reduced mean distance between vowels - i.e. a reduced degree of vowel disper- sion. These findings suggest that the overlap degree among vowels, not the vowel space size, is a better predictor of a speaker's overall intelligibility and, accordingly, of the severity of the speech disorder.

Our results also suggest that vowel contrasts, indexed by the overlap degree, are achieved not only by increased 
corner vowel space, but also by reduced variability in the vowel formants. Some speakers exhibited a trading relationship between these two modulations. Despite a considerable degree of $F_{1}$ variability, one speaker achieved a sufficient degree of distinctiveness among contrasting vowels through a substantially enlarged corner vowel space. On the other hand, one control speaker exhibited a rather compressed corner vowel space, even compared to moderate CP speakers, but the speaker maintained vowel distinctions through the precise articulation of each vowel token, as evidenced by the exceptionally small variability in $\mathrm{F}_{1}$ for each vowel phoneme. The trading relationship between vowel space size and $F_{1}$ variability demonstrates that the role of vowel space size in enhancing or reducing vowel phoneme distinctiveness can be influenced by formant variability. In other words, vowel space alone is not sufficient in addressing vowel characteristics and their relation to intelligibility. Based on our findings, the wide range of strength between vowel space areas and intelligibility reported in prior work on dysarthria can be attributed to the fact that a possible interaction of vowel space, formant variability and overlap degree was not considered.

Corner vowel space indicated a strong correlation with intelligibility at $\mathrm{r}^{2}=0.69$. This is considerably high compared to the previous findings of a speaking rate effect on vowel space and intelligibility [5-9]. A direct comparison with previous studies is impossible due to different methods and participant groups, but our finding suggests that a speaker's overall intelligibility and, accordingly, dysarthria severity is more strongly correlated to vowel space, compared to the intelligibility level induced by speaking rate modifications; further research is clearly required. The small effect of intelligibility on noncorner vowel space found in this study is similar to the findings on the PD group in the study by Tjaden et al. [14]. Clear speech findings were rather different [22-24]. For example, according to Picheny et al. [22], who compared the vowel space formed by the lax vowels $/ \mathrm{I}, \varepsilon, \Lambda$ / in clear versus conversational speech produced by normal speakers, the difference of vowel space area in clear versus conversational speech was greater for the noncorner vowel space than for the corner vowel space. Taken together, the results indicate that the extent to which intelligibility is related to vowel space may vary depending on vowel classes and depending on the type of speech (e.g. dysarthric vs. conversational normal vs. clear normal).

This study examined vowel formants in relation to overall speech intelligibility rather than vowel perception errors, concerning the relationship between vowel pro- duction and the severity level of dysarthria. It is yet to be discovered to what extent the acoustic correlates of vowels contribute to the intelligibility of various speech units (e.g. vowel phonemes, words, sentences and discourse). As Weismer et al. [3] and Weismer [25] discussed, a positive relation between vowel acoustics and speech intelligibility as found in this study leads to two contrasting hypotheses. The acoustic variables of vowels may be componential factors in overall intelligibility and thereby make a direct contribution to speech intelligibility levels. It is also possible that acoustic variables reflect overall speech mechanisms rather than a specific component of intelligibility. Aiming to test these hypotheses, future studies will have to include vowel perception error rates as well as overall intelligibility scores. Vowel perception data will also enable us to evaluate whether spastic dysarthria exhibits vowel error patterns similar to normal speech, or whether it manifests different error patterns due to the nature of the disorder. Finally, this study measured $F_{1}$ and $F_{2}$ values at the midpoint of the vowel steady state, capturing the targeting point of vowel production. Besides static measures, time-varying formant changes have been suggested to contribute to identifying vowels $[26,27]$. The role of formant dynamics was shown to be greater than the static measure, particularly in distinguishing vowels that are close in the vowel space (e.g. head vs. had). Future studies may thus extend to include formant measures at several time points and evaluate the extent to which dynamic versus static measures of formants are related to intelligibility in dysarthria.

\section{Conclusions}

The current study provided evidence for the relationship between intelligibility and the spectral characteristics of vowels produced by speakers with CP. Our results demonstrated that a speaker's overall intelligibility in this population was better represented by the overlap degree among vowels than by the vowel space. Speech pathologists may benefit from this result in their assessment of a speaker's intelligibility.

\section{Acknowledgments}

This study has been supported by an NIH grant (R21DC008090-A). The authors thank the subjects for their participation and Bowon Lee for building a microphone array. 


\section{References}

1 Turner GS, Tjaden K, Weismer G: The influence of speaking rate on vowel space and speech intelligibility for individuals with amyotrophic lateral sclerosis. J Speech Lang Hear Res 1995;38:1001-1013.

2 Liss LM, Spitzer SM, Cavinsess JN, Adler C, Edwards BW: Lexical boundary error analysis in hypokinetic and ataxic dysarthria. J Acoust Soc Am 2000;107:3415-3424.

$\checkmark 3$ Weismer G, Jeng JY, Laures JS, Kent RD, Kent JF: Acoustic and intelligibility characteristics of sentence production in neurogenic speech disorders. Folia Phoniatr Logop 2001;53:1-18.

$\checkmark 4$ Liu H, Tsao F, Kuhl PK: The effect of reduced vowel working space on speech intelligibility in Mandarin-speaking young adults with cerebral palsy. J Acoust Soc Am 2005;117: 3879-3889.

5 Tjaden K, Wilding GE: Rate and loudness manipulations in dysarthria: acoustic and perceptual findings. J Speech Lang Hear Res 2004;47:766-783.

6 Weismer G, Laures JS, Jeng JY, Kent RD, Kent JF: Effect of speaking rate manipulations on acoustic and perceptual aspects of dysarthria in amyotrophic lateral sclerosis. Folia Phoniatr Logop 2000;52:201-219.

$\checkmark 7$ Higgins CM, Hodge MM: Vowel area and intelligibility in children with and without dysarthria. J Med Speech Lang Pathol 2002; 10:271-277.

$\checkmark 8$ McRae PA, Tjaden K, Schoonings B: Acoustic and perceptual consequences of articulatory rate change in Parkinson's disease. J Speech Lang Hear Res 2002;45:35-50.

$\checkmark 9$ Hustad K, Lee J: Changes in speech production associated with alphabet supplementation. J Speech Lang Hear Res 2008;51:14381450
10 Smiljanic R, Bradlow AR: Production and perception of clear speech in Croatian and English. J Acoust Soc Am 2005;118:16771688.

11 Neel AT: Vowel space characteristics and vowel identification accuracy. J Speech Lang Hear Res 2008;51:574-585.

12 Ansel BM, Kent RD: Acoustic-phonetic contrasts and intelligibility in the dysarthria associated with mixed cerebral palsy. J Speech Hear Res 1992;35:296-308.

13 Bunton K, Weismer G: The relationship between perception and acoustics for a highlow vowel contrast produced by speakers with dysarthria. J Speech Lang Hear Res 2001;44:1215-1228.

14 Tjaden K, Rivera D, Wilding GE, Turner G: Characteristics of the lax vowel space in dysarthria. J Speech Lang Hear Res 2005;48: 554-566.

15 Kim H, Hasegawa-Johnson M, Perlman A, Gunderson J, Huang T, Watkin K, Frame S Dysarthric speech database for universal access research. Proceedings of the 2008 International Conference on Spoken Language Processing (ICSLP-Interspeech), Brisbane, 2008, pp 1741-1744.

16 Lee B, Hasegawa-Johnson M, Goudeseune C, Kamdar S, Borys S, Liu M: AVICAR: audiovisual speech corpus in a car environment. Proceedings of the 2004 International Conference on Spoken Language Processing (ICSLP-Interspeech), Jeju Island, 2004, pp 2489 2492.

17 Francis W, Kucera H: Frequency Analysis of English Usage. Lexicon and Grammar. Boston, Houghton Mifflin, 1982.
18 Hart MS: Project Gutenberg. 2005. http:// www.gutenberg.org/.

19 Whitehill TL: Assessing intelligibility in speakers with cleft palate: a critical review of the literature. Cleft Palate Craniofac J 2002; 39:50-58.

20 Hustad KC: Estimating the intelligibility of speakers with dysarthria. Folia Phoniatr Logop 2006;58:217-228.

21 Boersma P, Weenink D: Praat: doing phonetics by computer (version 4.4.24). 2006. http:// www.praat.org/.

22 Picheny MA, Durlach NI, Braida LD: Speaking clearly for the hard of hearing. 2. Acoustic characteristics of clear and conversational speech. J Speech Hear Res 1986;29: 434-446.

23 Bradlow AR, Kraus N, Hayes E: Speaking clearly for learning-impaired children: sentence perception in noise. J Speech Lang Hear Res 2003;46:80-97.

24 Smiljanic R, Bradlow AR: Clear speech intelligibility: listener and talker effects. Proceedings of the 2007 International Conference on Spoken Language Processing (ICSLP-Interspeech), Saarbrücken, 2007, pp 661-664

25 Weismer G: Speech intelligibility; in Ball MJ, Perkins MR, Müller N, Howard S (eds): Handbook of Clinical Linguistics. Malden, Wiley-Blackwell, 2008.

26 Huffman MK: Long Island vowels. 134th ASA meeting, San Diego, 1997. http://www. acoustics.org/press/134th/huffman.htm.

27 Assmann PF, Katz WF: Time-varying spectral change in the vowels of children and adults. J Acoust Soc Am 2000;108:18561866 